\title{
DEVELOPING A REAL TIME ALGORITHM FOR DIAGNOSING GLAUCOMA
}

\author{
Arwa Ahmed Gasm Elseid ${ }^{1}$, Alnazier Osman Hamza ${ }^{2}$ and Ahmed Fragoon ${ }^{3}$ \\ ${ }^{1,3}$ Department of Biomedical Engineering, Sudan University of Science and Technology, Sudan \\ ${ }^{2}$ Department of Radiology, University of Medical Sciences and Technology, Sudan
}

\begin{abstract}
A Glaucoma is a group of eye diseases causing optic nerve damage and if not detected at an early stage it may cause permanent blindness. Glaucoma progression precedes some structural damage to the retina are the symptoms of Glaucoma. Manually, it is diagnosed by examination of size, structure, shape, and color of optic disc and optic cup and retinal nerve fiber layer (RNFL), which suffer from the subjectivity of human due to experience, fatigue factor etc., and with the widespread of higher quality medical imaging techniques, there are increasing demands for computer-aided diagnosis (CAD) systems for glaucoma detection, because the human mistakes, other retinal diseases like Age-related Macular Degeneration (AMD) affecting in early glaucoma detection, and the existing medical devices like Optical Coherence Tomography (OCT) and Heidelberg Retinal Tomography (HRT) are expensive. This paper proposes a novel algorithm by extract 13 shape features from disc and cup, extract 25 texture features from RNFL(retinal nerve fiber layer) using gray level co-occurrence method and Tamara algorithm and 3 color features for each of disc and cup and RNFL. Next, best features selected using two methods, first method is the student t-test and the second method applied was the Sequential Feature Selection (SFS) to introduce the best 6 features. The evaluation of proposed algorithm is performed using a RIM_ONE and DRISHTI-GS databases, the average accuracy $97 \%$, maximize area under curve (AUC) 0.99, specificity $96.6 \%$ and sensitivity $98.4 \%$ using support vector machine classifier (SVM). Future works suggested to design a complete, automated system not just diagnose glaucoma but calculate the progress of the disease too.
\end{abstract}

Keywords:

Glaucoma, Fundus Image, Classification, GLCM Texture Feature, SFS

\section{INTRODUCTION}

Glaucoma is dangerous an ocular disease and a second cause of blindness in the world with about 60 million glaucomatous cases [1], and it is responsible for 5.2 million cases of blindness with more than $90 \%$ of the patients unaware of the condition mentioned [2] In 2014, the World Health Organization (WHO) reported that 285 million people were visually impaired and $90 \%$ of them lived in low-income at [3]. Clinically, in glaucoma, the optic nerve is progressively damaged with the disease progresses, and resultant in gradually vision loss and total blindness. As the optic nerve damage is irreversible, early detection of glaucoma is essential to prevent from blindness.

Currently, there are three methods for detecting glaucoma: assessment of the abnormal visual field, assessment of intraocular pressure (IOP) and assessment of optic nerve damage. Visual field test requires special equipment present only in hospitals and is a subjective examination needs fully understand the testing instructions, cooperate and complete the test. Moreover, and the information obtained may not be reliable for kids or ICU patient.
In the second method, a large proportion of glaucoma patients have a normal level of IOP. Thus, IOP measurement some timespecific nor sensitive for early glaucoma. The assessment of optic nerve damage is the best compared to the other two methods [4]. The optic nerve can be assessed by trained specialists or through 3D imaging techniques such as Heidelberg Retinal Tomography (HRT) and Ocular Computing Tomography (OCT). However, optic nerve assessment by specialists is subjective and the availability of HRT and OCT equipment is limited due to the high cost involved. In summary, there is still no systematic and economic way of detecting glaucoma. There is a need for automatic and economic system for detection of glaucoma in an accurate way, using the digital color fundus image. The Fig. 1 is more cost effective imaging modality to assess optic nerve damage compared to HRT and OCT, and it has been widely used in recent years to diagnose various ocular diseases, including glaucoma.

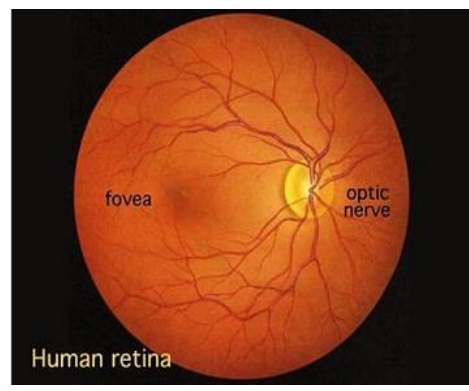

Fig.1. An example of fundus image obtained from fundus camera with a sign used for glaucoma detection [21]

Features extraction technique in fundus images are classified based on the type of features. The types of features are divided into two groups namely morphological and non-morphological [5], the morphological features based on optic disc and optic cup segmentation then calculate features like RNFL, CDR, PPA and ISNT rule as Mahalakshmi et al. [6] proposed a method to segment optic disc and optic cup using Simple Linear Iterative Clustering (SLIC) algorithm, K-Means clustering for glaucoma detection to obtain accurate boundary delineation. For optic disc and cup segmentation, the histograms and Centre statistics features were used to identify the disc part, the location information is also included into the feature space to boost the performance. Finally, the Cup to Disc Ratio (CDR) calculated for glaucoma screening as it is a primary identifier to confirm glaucoma for a patient.

Kausua [7] proposed a novel method for glaucoma identification based on time-invariant feature cup to disk ratio and anisotropic dual-tree complex wavelet transform features. Optic disk segmentation is done by using Fuzzy C-Means clustering method and Otsu's thresholding is used for optic cup segmentation. The obtained results achieved an accuracy rate of 
$97.67 \%$ with $98 \%$ sensitivity using a multilayer perceptron model. Babu et al. [8] proposed for the measurement of CDR as a sign for the diagnosis of glaucoma and $90 \%$ accuracy is obtained .Thus, it is an important indicator of the risk of the presence of glaucoma in an individual.

Yuji et al. [9] proposes a method to measure the cup-to-disc ratio using a vertical profile on the optic disc. First, the blood vessels were erased from the image and then the edge of optic disc was then detected by use of a canny edge detection filter. Then extract twenty profiles around disc center in the blue channel, and the profile was smoothed by averaging these profiles. After that, the cup area calculated by thresholding technique. Lastly, the CDR ratio was calculated using seventy-nine images, contain twenty-five glaucoma images, and obtained sensitivity of $80 \%$ and a specificity of $85 \%$.

Non-Morphological features are whole image features without segmentation like color, shape, and texture are type of the features that captured from the existing image. As Bock at [10] developed the previous research by modifying the feature extraction method used. The feature extraction methods were used such as the value of intensity pixel rows, FFT and B-spline methods, then reduced the size of the features dimensions using PCA. This research made the Glaucoma Risk Index (GRI) system using two-stage in the first stage classification combine different features (fraw, ffft, fspline), then in the second stage, the features normalized. The algorithm tested with 575 images (239 glaucoma and 336 normal images) and achieved accuracy of Area under Convergence (AUC) $80 \%$ and GRI $88 \%$.

The use of texture feature extraction has done by Karthikeyan [11] using two methods: histogram and Gray Level Cooccurrence Matrix (GLCM). The results of feature extraction were combined using the Sequential Forward Floating Selection (SFFS). Experimental fundus images obtained using the fundus camera Topcon TRC50 EX with image size $1900 \times 1600$ pixels derived from the Aravind eye hospital, Madurai. The results of the sensitivity, specificity, and accuracy reached respectively $96 \%$, $94 \%$ and $95 \%$ with 32 GLCM quantize level.

Therefore, based on ophthalmologist observation and retinal changes the paper proposes an automatic glaucoma detection method that uses the color, shape, texture feature in fundus images, with support vector machine (SVM) classifier. The aim of this method is to determine whether the fundus image of a patient is normal or glaucoma. Where the fundus camera is generally available in hospitals and eye care centers. Moreover, taking fundus images is inexpensive, and they can be used to check other diseases, such as diabetic retinopathy, hypertension, and myopia and can be used for the follow-up.

\section{MATERIAL AND METHOD}

Data Acquisition from An Open Retinal Image Database for Optic Nerve Evaluation (RIM-ONE) is a fundus image database that is related to glaucoma disease. It consists of $169 \mathrm{ONH}$ images, where the images are divided into several classes as follows: normal 118 images, early glaucoma 12 images, moderate glaucoma 14 images, deep glaucoma 14 images and ocular hypertension (OHT) 11 images. In this study, the RIM-ONE database is employed as it is considered precise gold standards of the ONH [12]. The Fig.2 demonstrates normal and abnormal glaucomatous images taken from the RIM-ONE database. Even if the images are clear, using the naked eye one cannot distinguish between the normal (healthy) images and the abnormal (glaucoma) images, which necessities the automated system development.

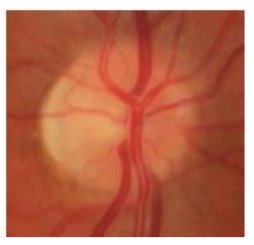

(a)

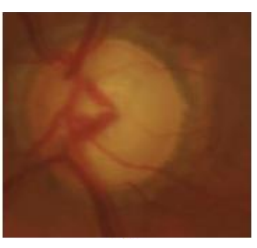

(b)
Fig.2. Eye RIM-ONE database images: (a) Normal image, (b) Glaucoma image (abnormal)

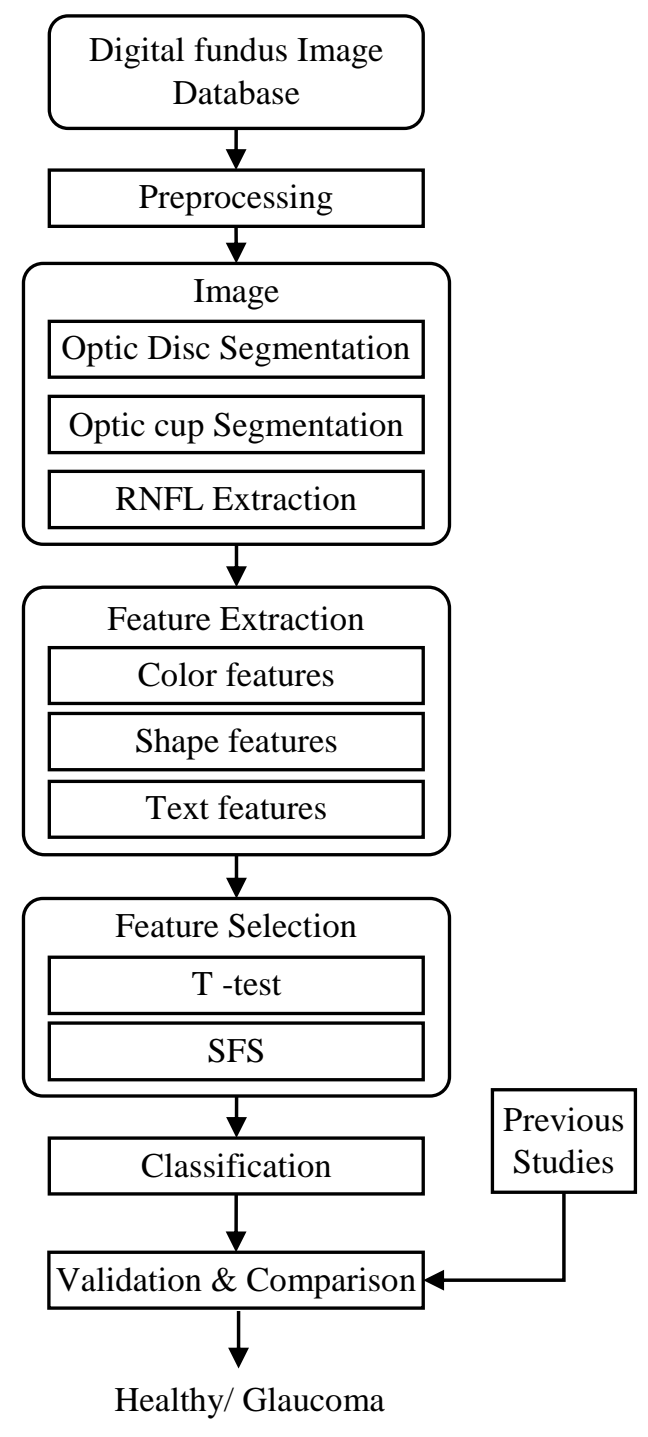

Fig.3. Proposed Methodology

Proposed algorithm provides an automated glaucoma detection computer-aided system that enables the ophthalmologists in early diagnosis of glaucoma patients with high accuracy. The algorithm takes a preprocessed fundus image and extracts optic cup and optic disc followed by the hybrid of features are extracted from the image to train and test the 
classifier. Result used to classify the image as glaucoma and healthy. The Fig.3 illustrates the complete methodology are explained in six steps: first step for preprocessing the interred fundus image, second step for OD, OC, and RNFL segmentation third section for features extraction where are color, shape and texture features based, forth step is features selection using t-test and SFS method to choose the features are quantitative measure for diagnosing glaucoma then, fifth step is the classification using the SVM classifier and finally compered the results with previous study to validate the system.

\subsection{PREPROCESSING STEP}

Image pre-processing processes involved are, resizing the image to $256 \times 256$ pixels so that it has the specified number of rows and column to reduce computational time, de noising stage by median filters $(5 \times 5)$. Before any procedures made, red channel has been extracted because it appears as good boundary and less blood vessel, which is affecting segmentation accuracy as to facilitate intensity analysis, as in Fig.4.

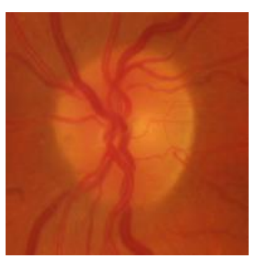

Original image

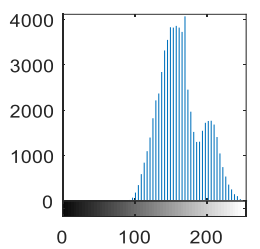

$0 \quad 100 \quad 200$

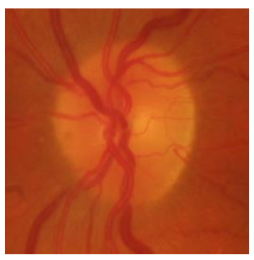

Resized image

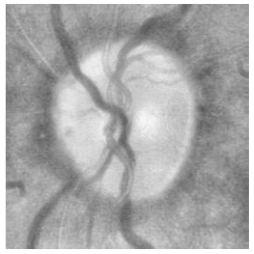

Adaptive

histogram

equalization image

Fig.4. Steps of an image preprocessing

\subsection{OPTIC DISC SEGMENTATION}

The Optic Disc Segmentation algorithm developed and tested in rim-one public database. The proposed method can be divided into 4 steps, first step is vessel remove to get accurate segmentation, and it done by morphological operation. Second step is applying thresholding level 180 to segment disc region from the red channel which appear best contrast to the disc region, after that in the third step is the boundary was smoothed and cleared from unconnected object and binary image obtained at the final step circle image was construct based on the radius and center of the detected region to minimize segmentation errors resulting from the main blood vessels and PPA surrounded the optic disc, using the formula and the result shown in Fig.5.

$$
(x-h)^{2}+(y-k)^{2}=r^{2}
$$

where $r=$ the radius from segmented object, $h, k=$ the center from segmented.
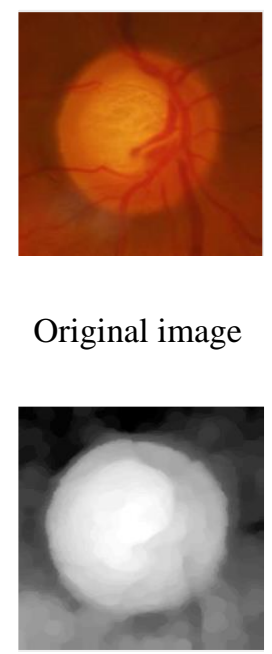

Histogram equalization

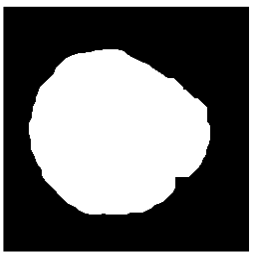

Clear border image
Original image

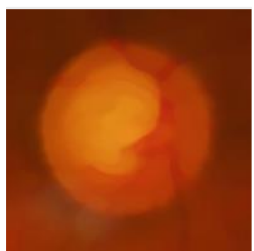

Vessel removed image

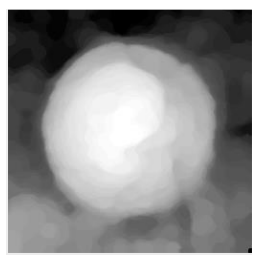

Filtered image

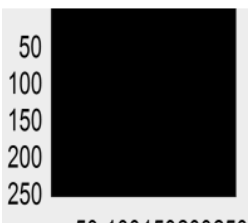

50100150200250

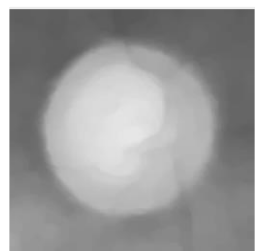

Red channel

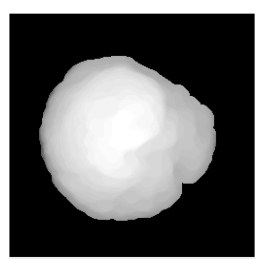

Segmented disc

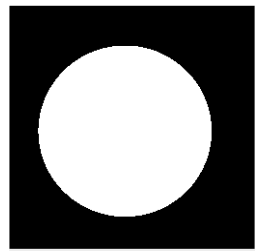

Constructed disc
Fig.5. Fundus image OD segmentation steps

\subsection{OPTIC CUP SEGMENTATION}

The optic cup segmentation method proposed, depend on optic disc thresholding at level 240, which is the best level differentiate between the disc and cup parts then clear border, smoothing boundary and binaries the final image,

Fixed thresholding is of the form: $O C(x, y)=\sum_{1}^{0} f(x, y)<t$ where $t$ is thresholding level $=240$.

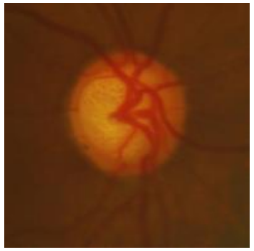

Original image

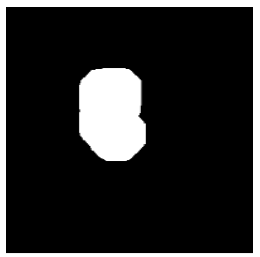

Segmented cup
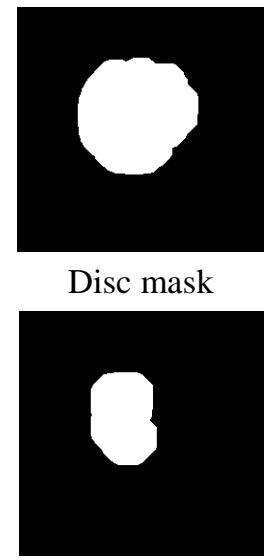

Cup mask
Disc mask

Fig.6. Cup segmentation and binarization from the disc part 


\subsection{RNFL ROI EXTRACTION}

The RNFL (retinal nerve fiber layer) is the area surrounded the disc for that the ROI determines based on mathematical method to subtract the whole image form disc area, to measure it is thickness, which affect by the glaucoma disease.

$R N F L=$ ROI region $-O D(3)$
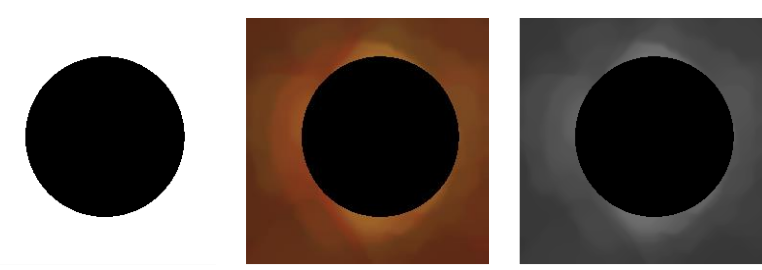

RNFL ROI

Original RNFL Gray RNFL

Fig.7. Shows RNFL for glaucoma image, which is apart surrounded the disc

\subsection{FEATURES EXTRACTION STEP}

Depending on ophthalmologist observation Glaucoma is diagnosed by examination of size, structure, shape and color of optic nerve head .Based on this theory a combination of color, structure, and shape features were applied to disc, cup and RNFL to classify glaucoma from non-glaucoma cases, the features are:

\subsubsection{Shape Features:}

Measures several properties describe shape for each disc and cup within an image via matlab built in function (region prop), Region props computes Area, Euler Number, Centroid, Filled Area, Filled Image, Extent, Major Axis Length, Minor Axis Length, Solidity and Perimeter, these measurements are applied for disc and cup masks.

\subsubsection{Texture Features:}

Several methods can be used to describe the main features of the textures such as coarseness and regularity. Gray-Level Cooccurrence matrices measure is one of the most important measures that can be used to describe the texture, in this paper two techniques used to describe the RNFL.

- GLCM Features: A statistical method of examining texture that considers the spatial relationship of pixels is the graylevel co-occurrence matrix (GLCM), also known as the gray-level spatial dependence matrix. The GLCM functions characterize the texture of an image by calculating how often pairs of pixel with specific values and in a specified spatial relationship occur in an image, creating a GLCM, and then extracting statistical measures which are Autocorrelation, Contrast, Correlation, Cluster Prominence, Cluster Shade, Dissimilarity, Energy, Entropy, Homogeneity, Maximum probability, Sum of squares, Sum average, Sum variance, Sum entropy, Difference variance, Difference entropy, Information measure of correlation1, Inverse difference (INV), Inverse difference normalized (INN), Inverse difference moment normalized ,some formula examples:

$$
\text { Contrast }=\operatorname{sum}_{i}\left(\operatorname{sum}_{j}\left((i-j)^{2} * p(i, j)\right)\right)
$$

Correlation $=\operatorname{sum}_{i}\left(\operatorname{sum}_{j}\left(\left(i-u_{i}\right)\left(j u_{j}\right) p(i, j) /\left(s_{i} \cdot s_{j}\right)\right)\right)$

$$
\begin{aligned}
& \text { Correlation }=\operatorname{sum}_{i}\left(\operatorname{sum}_{j}\left(\left((i j) p(i, j)-u_{x} \cdot u_{y}\right) /\left(s_{x} \cdot s_{y}\right)\right)\right. \\
& \operatorname{Energy~} \operatorname{sum}_{i}\left(\operatorname{sum}_{j}\left(p(i, j)^{2}\right)\right)
\end{aligned}
$$

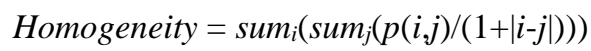

$$
\begin{aligned}
& \text { Homogeneity } \operatorname{sum}_{i}\left(\operatorname{sum}_{j}\left(p(i, \mathrm{j}) /\left(1+(i-j)^{2}\right)\right)\right)
\end{aligned}
$$

where:

$$
\begin{array}{r}
u_{i}=u_{x}=\operatorname{sum}_{i}\left(\operatorname{sum}_{j}(i \cdot p(i, j))\right) \\
u_{j}=u_{y}=\operatorname{sum}_{i}\left(\operatorname{sum}_{j}(j \cdot p(i, j))\right) \\
s_{i}=s_{x}=\operatorname{sum}_{i}\left(\operatorname{sum}_{j}\left(\left(i-u_{x}\right)^{2} \cdot p(i, j)\right)\right) \\
s_{j}=s_{y}=\operatorname{sum}_{i}\left(\operatorname{sum}_{j}\left(\left(j-u_{y}\right)^{2} \cdot p(i, j)\right)\right)
\end{array}
$$

where the values $i$ and $j$ are equal to 'NumLevels' parameter of the GLCM.

- Tamara Method: This method extracts the Tamura Features (Coarseness, Contrast, and Directionality) where:

Coarseness has a direct relationship to scale and repetition rates as the most fundamental texture feature. An image will contain textures at several scales; coarseness aims to identify the largest size at which a texture exists, even where a smaller micro texture exists. Computationally one first takes averages at every point over neighborhoods the linear size of which are powers of 2 . The average over the neighborhood of size $2 k \times 2 k$ at the point $(x, y)$ is:

$$
A_{k}(x, y)=\sum_{i=x-2^{k-1}-1}^{x+2^{k-1}-1} \sum_{j=y-2^{k-1}-1}^{i=y-2^{k-1}-1} \frac{f(i, j)}{2^{2 k}}
$$

Then at each point one takes difference between pairs of averages corresponding to non-overlapping neighborhoods on opposite sides of the point in both horizontal and vertical orientations. In the horizontal case this is

$$
E_{k, h}(x, y)=\left|A_{K}\left(x+2^{k-1}, y\right)-\left(x-2^{k-1}, y\right)\right|
$$

At each point, one then picks the best size which gives the highest output value, where $k$ maximizes $E$ in either direction. The coarseness measure is then the average of $\operatorname{spot}(x, y)=2 k_{\text {opt }}$ over the picture.

Contrast is a statistical distribution of the pixel intensity obtained. Rather, it is defined by the $\alpha_{4}=\mu_{4} / \sigma^{4}$ where $\mu_{4}$ is the fourth moment and $\sigma^{2}$ is the variance. Contrast is measured by the following formula:

$$
F_{c o n}=\frac{\sigma}{\alpha_{4}^{1 / 4}}
$$

This value gives the entire image or regions in contrast with global metrics.

Direction degrees we need to calculate the direction of the gradient vector is calculated at each pixel. And the direction of the vector mode are defined as:

$$
\begin{gathered}
|\Delta G|=\left(\left|\Delta_{H}\right|+\left|\Delta_{v}\right|\right) / 2 \\
\theta=\tan ^{-1}\left(\Delta_{v} / \Delta_{H}\right)+0.5 \pi
\end{gathered}
$$

where in $\Delta H$ and $\Delta V$ are the following two $4 \times 4$ operator variation resulting horizontal and vertical directions by the image convolution.

$$
\begin{aligned}
& \begin{array}{lllllll}
-1 & 0 & & 1 & 1 & 1 & 1
\end{array} \\
& \begin{array}{llllll}
-1 & 0 & 1 & 0 & 0 & 0
\end{array} \\
& \begin{array}{lllllll}
-1 & 0 & & 1 & -1 & -1 & -1
\end{array}
\end{aligned}
$$


When the gradient vector of all the pixels is calculated, a histogram is constructed for the expression of $H_{D} \theta$ value. The first range of values $\theta$ histograms were discrete, then the corresponding statistics for each bin of $|\Delta G|$ is greater than the number of pixels in a given threshold. The histogram of an image for a clear directional exhibit a peak, for no apparent direction of the image is relatively flat performance. Finally, the overall image can be calculated by the directional sharpness of peaks in the histogram obtained is expressed as follows:

$$
F_{d i r}=\sum_{p}^{n_{p}} \sum_{\varnothing \in \omega_{p}}\left(\theta-\theta_{p}\right) 2 H_{D}(\varnothing)
$$

where, $P$ represents the histogram of the peak type, $n p$ is the histogram of all the peaks. For a peak $p, W_{p}$ represents all peaks included in the bin, and the bin having the highest $\omega_{p}$ value.

\subsection{COLOR FEATURES}

Color moments are measures that characterize color distribution in an image in the same way that central moments uniquely describe a probability distribution. Color moments are mainly used for color indexing purposes as features in image retrieval applications in order to compare how similar two images are based on color. Two color moments are computed per channel, 6 color features are computed for each disc and cup.

Mean: The first color moment can be interpreted as the average color in the image, and it can be calculated by using the following formula:

$$
E_{i}=\sum_{j}^{N} 1 \frac{1}{N} p_{i j}
$$

where $N$ is the number of pixels in the image and $p_{i j}$ is the value of the $j^{\text {th }}$ pixel of the image at the $i^{\text {th }}$ color channel.

Standard Deviation: The second color moment is the standard deviation, which is obtained by taking the square root of the variance of the color distribution.

$$
\sigma_{i}=\sqrt{\left(\frac{1}{N} \sum_{j=1}^{N}\left(p_{i j}-E_{i}\right)^{2}\right)}
$$

where $E_{i}$ is the mean value, or first color moment, for the $i^{\text {th }}$ color channel of the image.

Skewness: The third color moment is the skewness. It measures how asymmetric the color distribution is, and thus it gives information about the shape of the color distribution (see section 2.8).

$$
S_{i}=\sqrt[3]{\left(\frac{1}{N} \sum_{j=1}^{N}\left(p_{i j}-E_{i}\right)^{3}\right)}
$$

\subsection{FEATURES SELECTION STEP}

Feature selection is the process of selecting a subset of relevant features for use in model construction.

The objective of this step is three-fold: improving the prediction performance of the predictors, providing faster and more cost-effective predictors, and providing a better understanding of the underlying process that generated the data.

In this paper two methods were applied.

\subsection{1 t-Test:}

t-Test applied with $p<0.05$ that result in 5 features, where a ttest's statistical significance used to compare the means of glaucoma and healthy samples.

\subsubsection{Sequential Feature Selection (SFS):}

SFS applied to the texture features get from the RNFL, where SFS is the simplest greedy search algorithm: Starting from the empty set, sequentially add the feature $x+$ that maximizes $\left(Y_{k}+x+\right)$ when combined with the features $Y_{k}$ that have already been selected SFS steps:

Step 1: Start with the empty set $Y_{0}=\{\varnothing\}$

Step 2: Select the next best feature $x+=\arg \max x \notin Y_{k} J Y_{k}+x$

Step 3: Update $Y_{k+1}=Y_{k}+x ; k=k+1$

Step 4: Goto 2

\subsection{CLASSIFICATION STEP}

At first many classification techniques are used like SVM, $\mathrm{KNN}$ and ensembles which use multiple learning algorithms to obtain better predictive performance than could be obtained from any of the constituent learning algorithms alone. To get more model accuracy Ensemble learning used to improve machine learning results by combining several models that is ensembles RUS Boost an excellent technique for learning from imbalanced data, the best accuracy was obtained by the SVM classifier.

\section{RESULTS AND DISCUSSION}

The performance of the proposed glaucoma detection method is evaluated in terms of sensitivity, specificity, accuracy and the ROC curve. Those values are defined as follows:

$$
\begin{gathered}
\text { Sensitivity }=T P /(T P+F N) \\
\text { Specificity }=T N /(F P+T N) \\
\text { Accuracy }=T P+T N /(T P+T N+F P+F N)
\end{gathered}
$$

The ROC curve shows true positive rate versus false positive rate for the currently selected trained classifier. You can select different classes to plot.

The marker on the plot shows the performance of the currently selected classifier. The marker shows the values of the false positive rate (FPR) and the true positive rate (TPR) for the currently selected classifier.

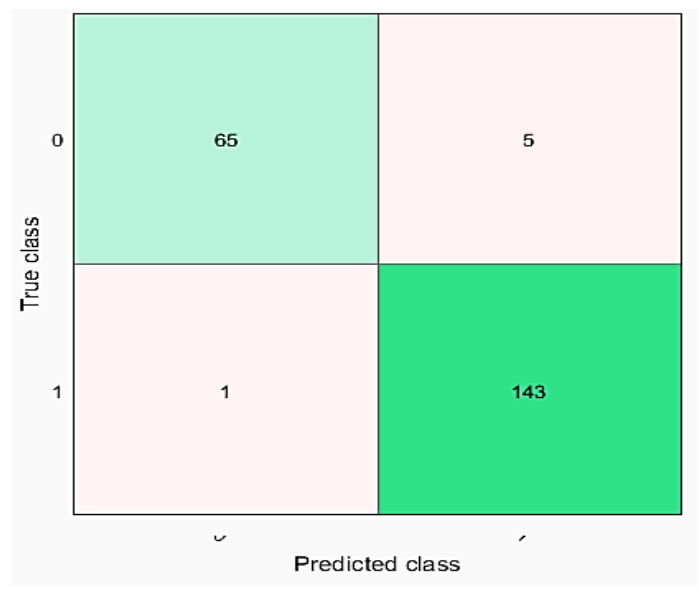




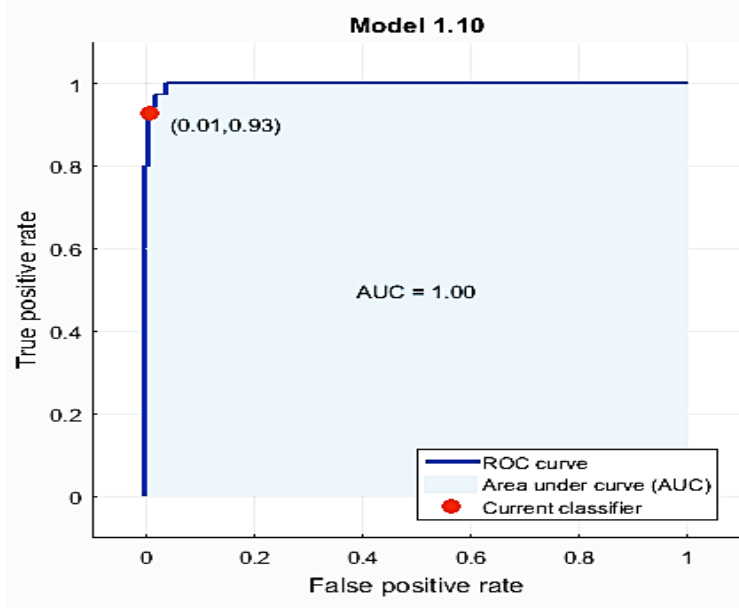

Fig.8. Shows confusion matrix and ROC curve results from the selected features

- True positive $(T P)=65$ is the number images detected as glaucoma by an expert and the proposed method.

- True negative $(T N)=143$ is the number of images detected as normal by an expert and the proposed method.

- False positive $(F P)=5$ is the number of images detected as normal by an expert but detected as glaucoma by the proposed method.

- False negative $(F N)=1$ is the number of images detected as glaucoma by an expert but detected as normal by the proposed method.

The values of sensitivity, specificity, and accuracy lie between 0 and 1. Therefore, if the result of the proposed method is accurate, it should be close to 1, the results shown in Table.1.

For more validation proposed algorithm compared with other glaucoma detection method used the same databases (RIM-ONE, DRISHTI-GS), the results shown in Table.2.
Table.1. Shows the final proposed system evaluation parameters values

\begin{tabular}{|c|c|c|c|}
\hline Sensitivity & Specificity & Overall accuracy & AUC \\
\hline $98.4 \%$ & $96.6 \%$ & $97 \%$ & $100 \%$ \\
\hline
\end{tabular}

From Table.2 noticed that [15] achieved 100\% accuracy, sensitivity and specificity using small database 13 healthy images 13 glaucoma images, and [13] get accuracy 98.5\% using twin support vector machine (TWSVM), where the TWSVM solves a pair of non-parallel hyper-planes whereas and [6] achieved accuracy rate of $97.67 \%$ with $98 \%$ sensitivity using a multilayer perceptron model classifier, where the SVM solves a single complex one and that prove the accuracy can further improved using different classifier or combined many classifiers together.

\section{CONCLUSION AND FUTURE WORK}

In conclusion, this paper proposed a glaucoma detection algorithm based on the analysis of fundus images fundus image, because it among one of the biomedical imaging techniques to analyze the internal structure of retina, using six features (cup minor axes, RNFL coarseness, disc mean and, standard deviation, and cup mean and standard deviation) classified by SVM. The proposed method achieved an accuracy of 97\%. The key contribution in this work is proposed features are suitable for glaucoma detection with high accuracy, the proposed method can make a valuable contribution to medical science by supporting medical image analysis for glaucoma detection via manage large volumes of data, provide objective assessments for decision support and help in labor intensive observer. The experimental results pointed out that the use of shape, color and texture features of correctly segment OC, OD and RNFL region are effected for glaucoma diagnosis. Future works suggested to: Designing a complete, integrated, automated system to classify all different types of glaucoma.

Complete the system to do not just diagnose glaucoma but compute the progress of the disease by comparing the different images of the same patient to be used for follow up.

Table.2. Comparisons of the proposed system with previous studies used the same databases

\begin{tabular}{|c|c|c|c|c|c|}
\hline Authors & Used Database & Features & Accuracy \% & Sensitivity $\%$ & Specificity \% \\
\hline Proposed system & DRISHTI-GSRIM-ONE & Color, shape and texture features & 97 & 98.4 & 96.6 \\
\hline Cheriguene [13] & RIM-ONE & TWSVM and three types of features & 98.53 & 94.11 & 100 \\
\hline Artem [14] & DRISHTI-GSRIM-ONE & U-Net Convolutional Neural Network & 92.08 & 80 & 62 \\
\hline Karkuzhali [15] & DRISHTI-GS & CDR,ISNT, DOO & 100 & 100 & 100 \\
\hline Sharanagouda [16] & DRISHTI-GS & CDR+ISNT & 96 & - & - \\
\hline Claro [17] & DRISHTI-GSRIM-ONE & Disc segmentation, texture feature & 93 & - & - \\
\hline Swapna [18] & DRISHTI-GS & $\begin{array}{l}\text { Texture Features Fractal Dimension + } \\
\text { LBP }\end{array}$ & 88.70 & 87.2 & 90 \\
\hline Arunava [19] & DRISHTI-GS & Segmentation, Image-based Features & 74.1 & 80 & 65 \\
\hline Ramaswamy [20] & RIM-ONE & A Depth Based Approach & - & 83 & 83 \\
\hline Maila [21] & DRISHTI-GSRIM-ONE & GLCM, Entropy & 93.03 & - & - \\
\hline Abir [22] & RIM-ONE & Grid Color Moment & 87.5 & - & - \\
\hline
\end{tabular}




\section{REFERENCES}

1. Paul N. Schacknow and John R. Samples, "The Glaucoma Book, A Practical Evidence-Based Approach to Patient Care", Springer, 2010.

2. Zhang Zhuo, Ruchir Srivastava, Huiying Liu, Xiangyu Chen, Lixin Duan, Damon Wing Kee Wong, Chee Keong Kwoh, Tien Yin Wong and Jiang Liu, "A Survey on Computer Aided Diagnosis for Ocular Diseases", BMC Medical Informatics and Decision Making, Vol. 17, No. 1, pp. 2-29 2014.

3. R. Koprowski, "Automatic Method of Analysis of OCT Images in Assessing the Severity Degree of Glaucoma and the Visual Field Loss", Biomedical Engineering, Vol. 13, No. 16, pp. 1-8, 2014.

4. Z. Zhang et al., "Automatic Glaucoma Diagnosis with mRMR-based Feature Selection", Biometrics and Biostatistics, Vol. 7, No. 8, pp. 1-8, 2012.

5. Anindita Septiarini and Agus Harjoko, "Automatic Glaucoma Detection based on the type of Features Used A review", Journal of Theoretical and Applied Information Technology, Vol. 72, No. 3, pp. 366-375, 2015.

6. V. Mahalakshmi and S. Karthikeyan, "Clustering Based Optic Disc and Optic Cup Segmentation for Glaucoma Detection", International Journal of Innovative Research in Computer and Communication Engineering, Vol. 2, No. 4, pp. 3756-3761, 2014.

7. Kausua Varun and P. Gopia Khan, "Combination of Clinical and Multi-Resolution Features for Glaucoma Detection and its Classification using Fundus Images", Biocybernetics and Biomedical Engineering, Vol. 38, No. 2, pp. 329-341,2018.

8. T.R. Babu and S. Shenbagadevi, "Automatic Detection Glaucoma using Fundus Image", European Journal of Scientist Research, Vol. 59, No. 1, pp. 22-32, 2011.

9. Yuji Hatanaka et al., "Vertical Cup-to-Disc Ratio Measurement for Diagnosis of Glaucoma on Fundus Images", Proceedings of International Conference on Computer Aided Design, pp. 1-8, 2010.

10. J. Bock et al., "Glaucoma Risk Index: Automated Glaucoma Detection from Color Fundus Images", Medical Image Analysis, Vol. 14, No. 3, pp. 471-481, 2010.

11. L. Karthikeyan and N. Rengarajan," Performance Analysis of Gray Level Co- Occurrence Matrix Texture Features for
Glaucoma Diagnosis", American Journal of Applied Sciences, Vol. 11, No. 2, pp. 248-257, 2014.

12. F. Fumero, S. Alayon and J.L. Sanchez, "An Open Retinal Image Database for Optic Nerve Evaluation", Proceedings of IEEE Symposium on Computer-Based Medical Systems, pp. 231-236, 2011.

13. N. Cheriguene et al., "New Computer Aided Diagnosis System for Glaucoma disease based on Twin Support Vector Machine", Proceedings of $1^{\text {st }}$ International Conference on Embedded and Distributed Systems, pp. 1236-1254, 2017.

14. Artem Sevastopolsky, "Optic Disc and Cup Segmentation Methods for Glaucoma Detection with Modification of UNet Convolutional Neural Network", Pattern Recognition and Image Analysis, Vol. 27, No. 3, pp. 618-624, 2017.

15. S. Karkuzhali and D. Manimegalai, "Computational Intelligence-based Decision Support System for Glaucoma Detection", Biomedical Research, Vol. 28, No. 11, pp. 4737 4748, 2017.

16. Sharanagouda Nawaldgi and Y.S. Lalitha, "A Novel Combined Color Channel and ISNT Rule Based Automatic Glaucoma Detection from Color Fundus Images", Indian Journal of Science and Technology, Vol. 10, No. 13, pp. 111-118, 2017.

17. M. Claro et al., "Automatic Glaucoma Detection Based on Optic Disc Segmentation and Texture Feature Extraction", Clei Electronic Journal, Vol. 19, No. 2, pp. 1-4, 2016.

18. P.P. Swapna and M.G. Mini, "A Regression Neural Network based Glaucoma Detection System using Texture Features", International Journal of Computing Communications and Instrumentation Engineering, Vol. 3, No. 2, pp. 1-7, 2016.

19. Arunava Chakravarty and Jayanthi Sivaswamy, "Glaucoma Classification with a Fusion of Segmentation and Imagebased Features", Proceedings of IEEE $13^{\text {th }}$ International Symposium on Biomedical Imaging, pp. 13-16, 2016.

20. A. Ramaswamy, K. Ram and M. Sivaprakasam, "A Depth Based Approach to Glaucoma Detection Using Retinal Fundus Images", Proceedings of International Workshop on Ophthalmic Medical Image Analysis, pp. 9-16, 2016.

21. Fundus Cameras, Available at: http://medical4help.blogspot.com/p/fundus-camera.html.

22. Abir Ghosh et al., "Grid Color Moment Features in Glaucoma Classification", International Journal of Advanced Computer Science and Applications, Vol. 6, No. 9, pp. 1-9, 2015. 